\title{
The Effects of PTK787/ZK222584, an Inhibitor of VEGFR and PDGFR $\beta$ Pathways, on Intussusceptive Angiogenesis and Glomerular Recovery from Thy 1.1 Nephritis
}

\author{
Monika Wnuk, ${ }^{*}$ Ruslan Hlushchuk, ${ }^{\dagger \ddagger}$ \\ Gérald Tuffin, ${ }^{*}$ Uyen Huynh-Do, * \\ and Valentin Djonov ${ }^{\dagger \neq}$

\begin{abstract}
From the Department of Nephrology and Hypertension,* Inselspital, University of Bern Medical School, Bern, Switzerland; the Anatomy Unit, ${ }^{\dagger}$ Department of Medicine, University of Fribourg, Fribourg, Switzerland; and the Institute of Anatomy,
\end{abstract} \\ University of Bern, Bern, Switzerland
}

The aim of our study was to investigate the phenomenon of intussusceptive angiogenesis with a focus on its molecular regulation by vascular endothelial growth factor receptor (VEGFR)/platelet-derived growth factor receptor $\beta$ (PDGFR $\beta$ ) pathways and biological significance for glomerular recovery after acute injury. Glomerular healing by intussusception was examined in a particular setting of Thy1.1 nephritis, where the lysis of mesangial cells results in an initial collapse and successive rebuilding of glomerular capillary structure. Restoration of capillary structure after induction of Thy1.1 nephritis occurred by intussusceptive angiogenesis resulting in i) rapid expansion of the capillary plexus with reinstatement of the glomerular filtration surface and ii) restoration of the archetypical glomerular vascular pattern. Glomerular capillaries of nephritic rats after combined VEGFR2 and PDGFR $\beta$ inhibition by PTK787/ZK222584 (PTK/ZK) were tortuous and irregular. However, the onset of intussusceptive angiogenesis was influenced only after long-term PTK/ZK treatment, providing an important insight into differential molecular regulation between sprouting and intussusceptive angiogenesis. PTK/ZK treatment abolished $\alpha$-smooth muscle actin and tensin expression by injured mesangial cells, impaired glomerular filtration of microspheres, and led to the reduction of glomerular volume and the presence of multiple hemorrhages detectable in the tubular system. Collectively, treatment of nephritic patients with PTK/ZK compound is not recommended. (Am J Patbol 2011, 178:1899-1912; DOI: 10.1016/j.ajpath.2010.12.049)

The concept of intussusceptive angiogenesis, an alternative to sprouting mode of angiogenesis, was postulated two decades ago within the rapidly expanding pulmonary capillary bed of neonatal rats. Numerous slender intraluminal tissue pillars, the hallmarks of intussusception, were observed in the lung capillaries. ${ }^{1,2}$ It was postulated that the pulmonary capillary network expands predominantly by the insertion of transcapillary pillars, a phenomenon termed intussusceptive microvascular growth, thereby implying that the capillary network expanded "within itself."

Further investigations demonstrated that intussusception is an important mechanism of vascular growth, remodeling, and angioadaptation. It permits a rapid expansion of the capillary plexus (intussusceptive microvascular growth), plays an essential role in the segregation of small arteries and veins (intussusceptive arborization), and optimizes the branching geometry to changes in blood flow (intussusceptive branching remodeling). Intussusception can also lead to the degeneration of a vessel branch; this process is termed intussusceptive pruning (for more details see reviews by Djonov and coworkers). ${ }^{3-5}$ Recently, the presence of intussusceptive angiogenesis was demonstrated during kidney and lung development in chickens. It has been confirmed that the primary capillary plexus is formed, as hitherto believed, by sprouting angiogenesis but that subsequent vascular growth and, most importantly, the formation of an organ-specific angioarchitecture occur mainly by intussusception. Compared with sprouting, intussusceptive angiogenesis is faster and does not require extensive endothelial cell proliferation and the accompanying vascu-

Supported by grant 3100A0-1183691 of the Swiss National Science Foundation to Uyen Huynh-Do and grant 31003A-116243 of the Swiss National Science Foundation to V.D.

Compound PTK787/ZK222584 was provided by Novartis Pharma AG (Basel, Switzerland)

U.H.-D. and V.D. contributed equally to this work.

Accepted for publication December 30, 2010.

Supplemental material for this article can be found at http://ajp. amjpathol.org or at doi: 10.1016/j.ajpath.2010.12.049

Current address of M.W.: Institute of Anatomy, University of Bern, Bern, Switzerland; of G.T.: Glycart Biotechnology, Wagistrasse 18, Schlieren, Switzerland

Address reprint requests to Valentin Djonov, M.D., Institute of Anatomy, University of Bern, Baltzerstrasse $2 \mathrm{CH}-3009$ Bern, Switzerland. E-mail: valentin.djonov@ana.unibe.ch. 
lar permeability remains low (ie, on a physiologic level). As a result, the vasculature could expand without compromising the specific functions of the organ. Importantly, only sprouting angiogenesis can vascularize avascular regions, whereas intussusception acts merely in preexisting capillary plexuses. ${ }^{6,7}$

Thy 1.1 nephritis is a well-established model for studying restorative remodeling of glomerular structure after acute injury. Administration of an anti-Thy1.1 antibody causes transient mesangial and successive vascular injury, but glomerular function and capillary structure are completely restored in approximately 3 to 4 weeks. ${ }^{8}$ Endothelial and mesangial regeneration resulting in the capillary growth and rebuilding of the glomerular angioarchitecture is an essential step in the repair process. 9,10 Recently, Notoya and coworkers ${ }^{11}$ demonstrated that intussusceptive angiogenesis is involved in the process of Thy1.1 nephritis recovery. Combining different morphologic approaches, the authors showed that formation of transluminal tissue pillars is involved in the postinjury glomerular angiogenesis. They suggested that a critical role is played by endothelial and mesangial cells in this process. Initially, the endothelial cells build the pillars, which are subsequently stabilized by cytoplasmic protrusions of mesangial cells in the vicinity of the latter. The active role of mesangial cells in the pillar formation has been recently hypothesized by Ichimura and colleagues. ${ }^{12}$ The authors demonstrated the transient mesangial expression pattern of $\alpha$-smooth muscle actin ( $\alpha$ SMA) during the recovery period and suggested that contraction of $\alpha$-SMA-positive mesangial protrusions within the pillars contributed to intussusceptive angiogenesis and normalization of the glomerular volume.

We have previously shown that in tumors treated with PTK787/ZK222584 (PTK/ZK), a small-molecular-weight inhibitor of vascular endothelial growth factor receptor (VEGFR) and platelet-derived growth factor receptor $\beta$ (PDGFR $\beta$ ) signaling, ${ }^{13}$ intussusception is the angiogenic mechanism that permits tumor regrowth. ${ }^{14}$

Because VEGFRs and PDGFR $\beta$ are by far one of the most relevant receptor tyrosine kinases for endothelial and pericyte functions, respectively, ${ }^{15}$ we asked whether PTK/ZK administration affects intussusceptive vessel splitting in a particular setting of physiologic organ recovery, namely, in kidney recovery from Thy 1.1 nephritis. We have also followed the effects of PTK/ZK on mesangial biology and glomerular permeability in nephritic glomeruli.

\section{Materials and Methods}

\section{Animals and Experimental Protocols}

All procedures were conducted according to the National Institutes of Health guidelines for the care and use of laboratory animals and with the approval of the local animal ethics committee.

Male Wistar rats weighing 170 to $190 \mathrm{~g}$ at the beginning of the study were obtained from Charles River Laboratories, Sulzfeld, Germany.
Acute Thy1.1 glomerulonephritis was induced by a single intravenous injection of $1 \mathrm{mg} / \mathrm{kg}$ of monoclonal OX7 antibody. ${ }^{16}$ Rats were sacrificed on days $3,5,9,14$, and 21 . Animals receiving the same volume of saline served as control.

\section{PTK/ZK Experiments}

PTK/ZK was obtained from Novartis Pharma AG, Basel, Switzerland. A total of $1 \mathrm{mg} / \mathrm{kg}$ of monoclonal OX7 antibody was injected to the rat tail vein. OX7 binds to the Thy 1.1 molecule within the first 2 hours after injection. ${ }^{16}$ Administration of PTK/ZK began a few hours after OX7 injection to avoid potential influence of the compound on OX7 binding to Thy1.1 molecule. PTK/ZK was dissolved in PEG300 at a concentration of $20 \mathrm{mg} / \mathrm{mL}$. Animals received $50 \mathrm{mg} / \mathrm{kg}$ of PTK/ZK intraperitoneal injection once daily. Control nephritic animals received PEG300 alone. Compound/vehicle administration continued for $5,9,14$, or 21 days. At the end of the experiment, kidneys were prepared for further analysis.

\section{Primary Antibodies}

Anti-VEGFR2 and anti-phospho-PDGFR $\beta$ (Tyr716) antibodies were obtained from Millipore (Bedford, MA). Anti$\beta$-actin and antitensin antibodies were from Santa Cruz Biotechnology (Santa Cruz, CA). Antibodies against protein kinase B (PKB), phospho-PKB (Ser473), and phospho-VEGFR2 (Tyr1175) were purchased from Cell Signaling Technology (Danvers, MA). Anti- $\alpha$-SMA antibody was from Sigma (St. Louise, MO), anti Ki-67 was obtained from Dako (Glostrup, Denmark), and antisynaptopodin from Synaptic Systems (Goettingen, Germany). Antibodies against the following proteins were purchased from Abcam (Cambridge, UK): PDGFR $\beta$, VEGF, VEGFR1, and phosphoVEGFR1 (Tyr1213). Anti-CD31 antibody was from Abcam or BD Biosciences (Heidelberg, Germany).

\section{Light Microscopy, Scanning Electron Microscopy on Tissues Dried at Critical Point, and Immunohistochemistry}

For light microscopy on semithin sections, kidney pieces were fixed in $2.5 \%$ glutaraldehyde in $0.1 \mathrm{M}$ cacodylate buffer ( $\mathrm{pH}$ 7.4, $350 \mathrm{mOsm}$ ). Tissue blocks were postfixed in osmium tetroxide, block stained using uranyl acetate, dehydrated through ascending concentrations of ethanol, and embedded in epoxy resin. Semithin sections were obtained at a nominal thickness of $1 \mu \mathrm{m}$, and stained with toluidin blue.

Kidney tissue fixed in the same way was used for the preparation of the specimens dried at critical point. Tissue pieces were cut into 1- to 2-mm sections and were subsequently immersed into the same fixative as used for perfusion. Probes were then washed, dehydrated in ascending concentrations of ethanol, and dried in a desiccator. Samples were mounted on aluminum stabs, sputter-coated with gold, and viewed with scanning electron microscopy (SEM) (Philips XL 30, Eindhoven, The Netherlands). 
Kidney tissue for immunohistochemical analysis was perfusion fixed with $2 \%$ paraformaldehyde, embedded in paraffin, and sectioned at $5 \mu \mathrm{m}$. A signal amplification kit based on streptavidin-biotin-peroxidase reaction (CSA TM; Dako) was used to visualize VEGFR2 and VEGF according to the manufacturer's instructions. Control experiments included omission of the primary antibody and substitution of the primary antibody with nonimmune serum.

Images were obtained with a Nikon Eclipse E600 microscope (Nikon, Tokyo, Japan) and captured with the Nikon digital camera DXM1200.

\section{Confocal Laser Scanning Microscopy}

Indirect immunofluorescence staining was performed with cryosections $(5 \mu \mathrm{m})$ that had been postfixed in ice-cold methanol:acetone (1:1 vol/vol) for 10 minutes and air dried. Sections were incubated with primary antibody overnight at $4^{\circ} \mathrm{C}$. Bound antibody was visualized using Alexa Fluor 488 goat anti-rabbit IgG or Alexa Fluor 594 goat anti-mouse lgG (Invitrogen, Karlsruhe, Germany) (30 minutes, room temperature). Sections were evaluated with laser scanning microscopy (LSM510; Carl Zeiss, Heidelberg, Germany).

\section{Studies of Glomerular Permeability with Fluorescent Microspheres}

At day 5 of nephritis, rats received intravenous injection of $600 \mu \mathrm{L}$ of FluoroSpheres carboxylate-modified microspheres, red fluorescent 580/605 (Invitrogen). The diameter of the microspheres was $100 \mathrm{~nm}$. Animals were sacrificed 30 minutes later, and kidneys were frozen for further analysis. Cryosections were stained with different markers of glomerular cells in green, counterstained with DAPI, and evaluated with laser scanning microscopy.

\section{Glomeruli Isolation and Western Blotting}

Rat glomeruli were isolated using the standard sieving method. Isolated glomeruli were homogenized in lysis buffer containing $50 \mathrm{mmol} / \mathrm{L}$ Tris- $\mathrm{HCl}(\mathrm{pH} 7.2), 120$ $\mathrm{mmol} / \mathrm{L} \mathrm{NaCl}, 1 \mathrm{mmol} / \mathrm{L}$ EDTA (pH 8.0), $6 \mathrm{mmol} / \mathrm{L}$ ethyleneglycoltetracetic acid ( $\mathrm{pH}$ 8.5), $1 \%$ Nonidet-P 40 , and $20 \mathrm{mmol} / \mathrm{L} \mathrm{NaF}$; lysis buffer was freshly supplemented with $0.1 \% \mathrm{SDS}, 2 \mathrm{mmol} / \mathrm{L}$ orthovanadate, $10 \mathrm{mmol} / \mathrm{L}$ sodium pyrophosphate, and protease inhibitors cocktail tablet (Roche Diagnostics, Basel, Switzerland). Lysates were centrifuged at $15,000 \times g$ for 30 minutes at $4^{\circ} \mathrm{C}$ and supernatant was collected for further analysis. SDS polyacrylamide gel electrophoresis was performed with 30 to $100 \mu \mathrm{g}$ of glomerular proteins.

Samples were run under reducing conditions on SDS polyacrylamide gel and transferred onto polyvinylidene difluoride membrane (Millipore). In case of Thy 1.1 detection with OX7 antibody, the samples were run under nonreducing conditions. Membranes were blocked in blocking buffer ( $5 \%$ nonfat dry milk in Tris-buffered saline, $0.1 \%$ Tween 20 ) and incubated overnight at $4{ }^{\circ} \mathrm{C}$ with primary antibody. Peroxidase-conjugated goat antimouse $(1: 10,000)$ and goat anti-rabbit $(1: 20,000)$ were used as secondary antibodies (Santa Cruz Biotechnology). Membranes were stripped with $0.1 \mathrm{M}$ glycine $(\mathrm{pH}$ 2.5) when indicated and reprobed with the appropriate antibody. Western blots were quantified with ImageJ software (http://rsbweb.nih.gov/ij/index.html). To quantify the effects of PTK/ZK on receptor phosphorylation, first the expression of phosphorylated and total receptor was adjusted to the $\beta$-actin expression. Next, the expression of phosphorylated and total receptor was quantified, indicating the percentage of phosphorylated receptor. Because PTK/ZK affected not only phosphorylation status but also expression of the receptor, the ratio was next multiplied by the total amount of receptor. In this way a value named "amount of phosphorylated receptor" was obtained.

\section{Vascular Casting and Morphometric Analyses}

For the preparation of vascular casts, systemic vasculature was perfused with a freshly prepared Mercox solution (Vilene Company, Tokyo, Japan) containing $0.1 \mathrm{~mL}$ of accelerator per $5 \mathrm{~mL}$ of resin. When resin polymerized, kidneys were excised and transferred to $7.5 \%$ potassium hydroxide for the dissolution of surrounding not perfused tissue.

Pictures of glomeruli were taken at $\times 1200$ magnification with SEM (Philips XL 30) to assess the density of tiny holes and small capillary loops. At least 50 glomeruli from each animal were used for quantification. The image analysis was performed with iTEM software (Olympus, Hamburg, Germany). Pillars were identified in the vascular casts as tiny holes whose larger diameter was lesser than $2 \mu \mathrm{m}$. All tiny holes whose larger diameter was between 2 and $10 \mu \mathrm{m}$ were considered as small capillary loop. ${ }^{11}$ Tiny hole and small capillary loop appearance was expressed as numerical density per vessel area.

Glomeruli volume $\left(V_{G}\right)$ was quantified from SEM pictures of vascular casts. It was assumed that glomeruli are ellipsoidal; therefore, the following formula was used to calculate the mean glomerular volume:

$$
V_{G}=(4 / 3) \times \pi \times(L / 2) \times(I / 2) \times(I / 2)
$$

where $\pi=3.1416, \mathrm{~L}$ is the length of longest glomerular diameter, and I is the length of the diameter perpendicular to the longest one. At least 100 glomeruli from each animal were used for quantification.

The total number of glomeruli in the left kidney was determined by the physical dissector/fractionator stereologic technique. ${ }^{17}$

Microaneurysm appearance was quantified from scanning electron micrographs. A total of 100 glomeruli were randomly chosen, and the diameter of enlarged vessels was measured. Dilated vessel was selected as microaneurysm if its diameter exceeded $50 \mu \mathrm{m}$.

\section{Urinanalysis}

For the assessment of proteinuria, rats were housed individually in metabolic cages for collection of 24-hour urine specimens. Urinary proteins were precipitated with 30\% trifluoroacetic acid, and protein pellet was resuspended in $0.25 \mathrm{~N} \mathrm{NaOH} .{ }^{18}$ Protein concentration was determined with 
a bicinchoninic acid kit (Thermo Fischer Scientific, Rockford, IL). Bovine serum albumin diluted in $0.25 \mathrm{~N} \mathrm{NaOH}$ served as standard.

\section{Statistical Analyses}

All data presented are expressed as mean \pm SD. Statistical analyses were performed with GraphPad Prism software (La Jolla, CA). An unpaired $t$-test was used when single comparisons were performed. For multiple comparisons, one-way analysis of variance with an appropriate post hoc test was used. Results at $P<0.05$ were considered significant.

\section{Results}

\section{Thy1.1 Nephritis Recovery Is Associated with Intussusceptive Angiogenesis, Increase of Glomerular Volume, and Loss of Nephrons}

To determine the progress of glomerular revascularization after OX7 injection in rats, animals were sacrificed at days $3,5,9,14$, and 21 of nephritis. We used the vascular casting method to visualize the vascular damage and three-dimensional alterations in the capillary structure on nephritis induction.

Glomerular capillaries of control animals assemble in a compact, uniform, classic pattern with rather large intercapillary distance. The signs of intussusceptive remodeling (eg, transluminal tissue pillars represented by holes in the casting material) are not frequent in healthy glomeruli (Figure 1 A). At day 3 of nephritis, glomerular vasculature is severely destroyed. It appears disrupted, and only peripheral capillaries close to the vascular pole are perfused (Figure 1B, asterisks). Day 5 of nephritis is characterized by enlarged capillaries with diameters greater than $50 \mu \mathrm{m}$, termed microaneurysms herein (Figure 1, C and D, asterisks). The presence of microaneurysms is of a temporary nature, and by day 9 of nephritis their occurrence decreased more than eight times, by day 14 their occurrence decreased by more than 30-fold, and at day 21 they are not detectable (see Supplemental Figure S1A at http://ajp.amjpathol.org). From day 5 of nephritis on, vascular casts revealed the presence of tiny holes in glomerular capillaries (Figure 1, D, F, H, and $J$, arrowheads), which are the hallmarks of intussusceptive angiogenesis. It seems that, on the one hand, the newly formed pillars separate and slash the microaneurysms (asterisk in Figure 1C), and on the other hand they serve as a material for vascular splitting. The presence of tiny holes was even more pronounced on days 9 (Figure 1, E and F) and 14 (Figure 1, G and H) of nephritis. Intussusception was still taking place at day 21 of nephritis (Figure 1, I and J). After the transluminal tissue pillars were formed, they next increased in girth, enlarged, and became capillary loops (Figure $1 \mathrm{H}$, arrows). We could not observe any sprouts arising from glomerular capillaries.

The phenomenon of pillar insertion and enlargement contributes to the rapid growth of glomerular vasculature. At days 9,14 , and 21 we noticed that pillars and small capillary loops often arrange themselves in rows. This
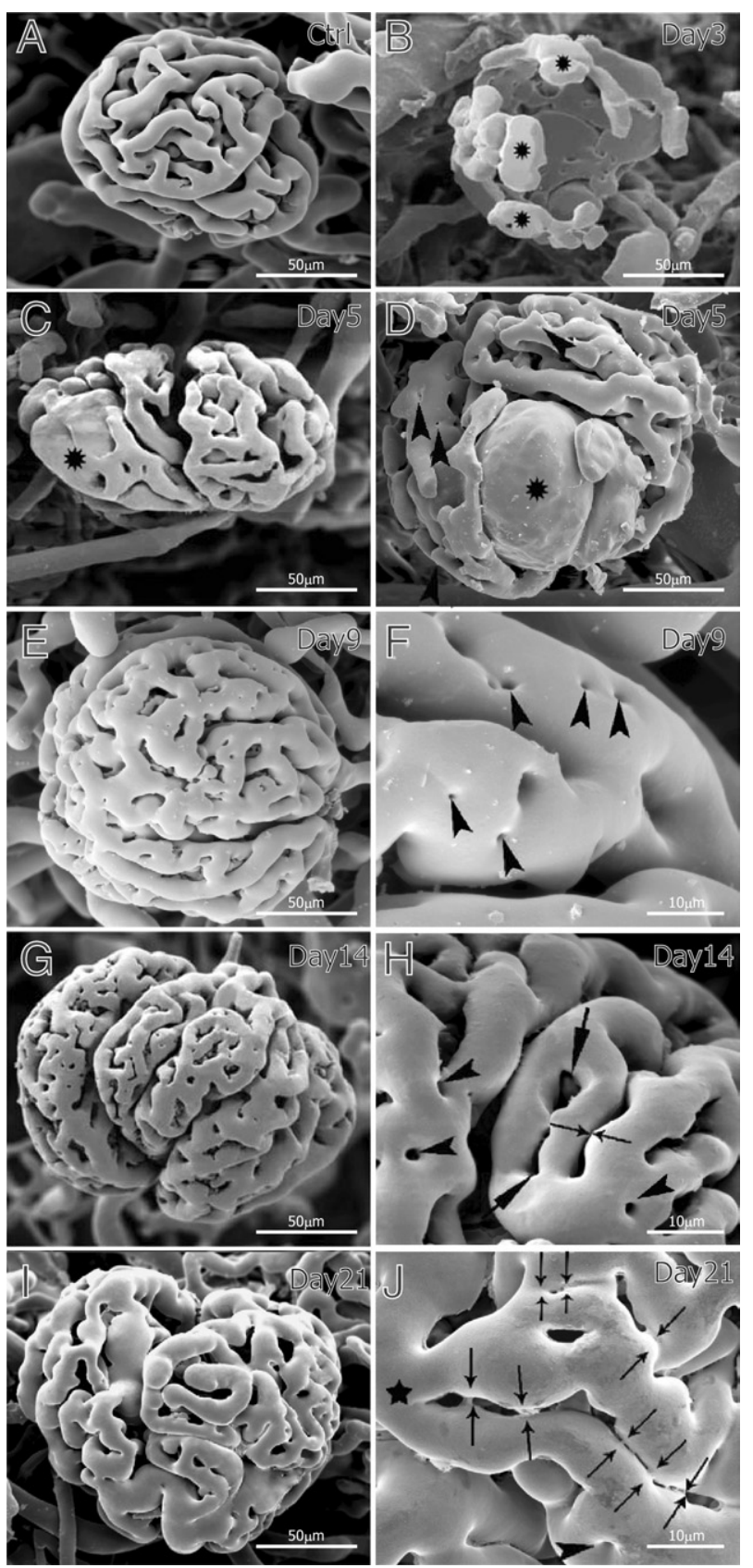

Figure 1. Corrosion vascular casts of Thy1.1 nephritic kidneys. The control group (A) is characterized by glomeruli with slender capillaries and wide intercapillary spaces. At day 3 of nephritis (B), the glomerular vasculature is destroyed and only the peripheral vessels close to the vascular pole are preserved (asterisks). On day 5 ( C and $\mathbf{D})$, enlarged glomeruli containing microaneurysms (asterisks) are frequently observed and tiny holes start to be clearly detected (arrowheads in $\mathbf{D})$. At day $9(\mathbf{E}$ and $\mathbf{F})$, density of pillars (arrowheads in F) increased remarkably and many arose in rows. At day 14 $(\mathbf{G}$ and $\mathbf{H})$, pillars (arrowheads in $\mathbf{H})$ enlarged in girth, merged, and formed small capillary loops (arrows in $\mathbf{H})$. Starting from day $14(\mathbf{G}$ and $\mathbf{H})$, and at day 21 (I and $\mathbf{J}$ ), the capillary loops ( $\mathbf{H}$, arrows) are often arranged in rows, indicating the advanced stage of capillary splitting. $\mathbf{H}$ and $\mathbf{J}$ : Thin arrows depict breaking connections and successive vessel splitting of the mother (main) segment (J, asterisk)

route of development is involved in rebuilding of the glomerular specific angioarchitecture. The process started by an arrangement of pillars in rows along the long axis of the blood flow, thus delineating a new vascular entity (Figure 1F, arrowheads). Subsequently, the pillars re- 
shaped, became elongated (arrows in Figure 1H), and merged with the neighboring ones, forming slits. Successive attenuation of the connecting bridges (thin arrows in Figure 1, $\mathrm{H}$ and $\mathrm{J}$ ) split the main vessel (Figure 1J, black asterisk) and segregated solitary capillary segments from the uniform capillary plexus. At day 21 , the process was rather advanced, and the typical glomerular vessel angioarchitecture was almost rebuilt (Figure 1, I and J). Intussusceptive angiogenesis is thus not only restoring capillary volume and number but also reestablishing the archetypical glomerular vascular pattern. The quantification of tiny holes and small capillary loops in the vascular casts of nephritic glomeruli as numerical density per vessel area confirmed the dynamic nature of intussusceptive remodeling during nephritis recovery (Figure 2A). Starting from day 5 , the number of pillars increased with a peak of intussusception at day 14 . As the number of tiny holes increased, the number of small capillary loops increased concomitantly and the entire glomerulus expanded progressively. The onset of intussusception decreased at day 21 of nephritis, when the glomerular angioarchitecture is almost restored. The number of glomerular proliferating cells was highest at days 3 and 5 of nephritis and gradually decreased (Figure 2B), thus showing inversed dynamics compared with intussusception. Morphometric analysis of vascular casts showed that glomerular volume increased significantly on nephritis induction (Figure 2C). After a peak at day 5, the volume started to decrease but did not reach the basal level. At day 21 , glomeruli remained on average $65 \%$ larger than in the control group (control: $841 \times 10^{3} \mu \mathrm{m}^{3} \pm 18 \times 10^{3} \mu \mathrm{m}^{3}$; day 21 of nephritis: $1387 \times 10^{3} \mu \mathrm{m}^{3} \pm 442 \times 10^{3} \mu \mathrm{m}^{3}$ ), although the difference was not statistically significant.

The course of acute mesangioproliferative nephritis led to a decrease in the number of nephrons (Figure 2D). Stereologic evaluation of the glomerular number showed that there was $39 \%$ less glomeruli at day 21 of nephritis $\left(18.9 \times 10^{3} \pm 0.6 \times 10^{3}\right)$ compared with healthy controls $\left(31 \times 10^{3} \pm 1.8 \times 10^{3}\right)$.

\section{Semithin Sectioning of Healthy and Nephritic Kidney Depicts the Progress of Glomerular Recovery and Intussusception Onset}

The semithin sectioning of nephritic kidneys harvested at different time points allowed us to follow the changes in glomerular morphology on nephritis induction. Capillaries of healthy glomerulus present a uniform structure with inconspicuous mesangial region (Figure 3A). Glomerular vasculature was severely reduced at day 3 of nephritis, causing enlargement of the urinary space (Figure 3B, white asterisks). The first microaneurysms were already detectable (Figure 3B, black asterisk). From day 5 on (Figure 3C), a compact mesangial region (white asterisk) was visible beside the enlarged capillaries (black asterisks) and microaneurysms were filled with erythrocytes. At day 9 of nephritis, the mesangial region was represented by a dense mesangial area (Figure 3D, white asterisk), pushing the capillaries toward the glomerular periphery (Figure 3D, black asterisks). At day 14 , we noticed the presence of thin
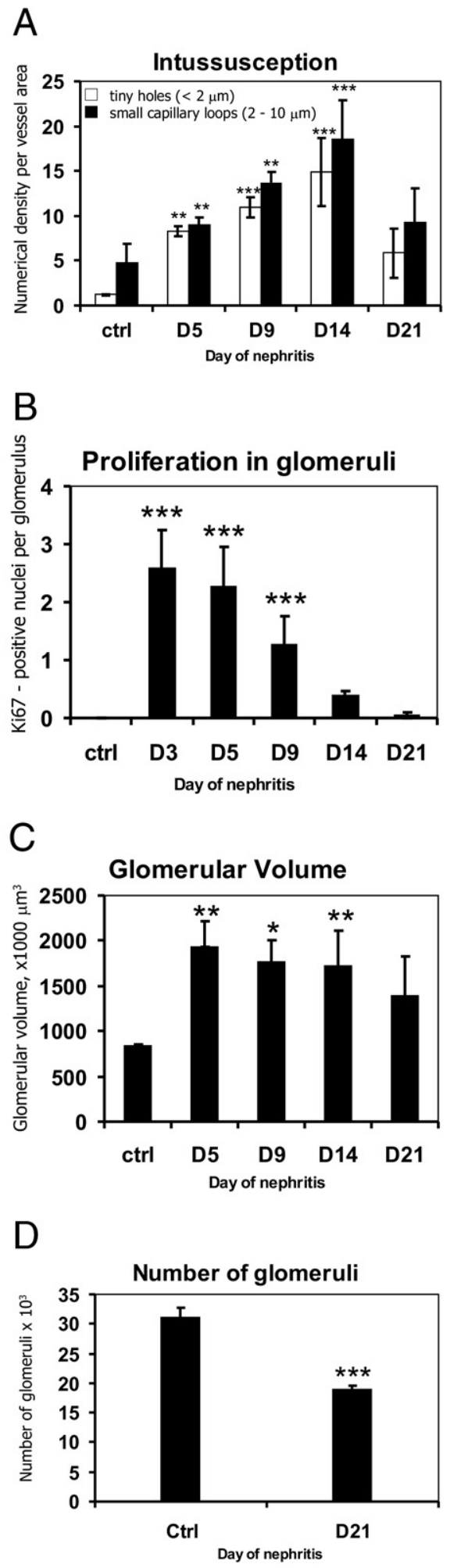

Figure 2. Morphometric analysis of glomerular intussusception, proliferation, volume, and number throughout Thy1.1 nephritis course. Quantification of pillar and capillary loop density at different time points showed the involvement of intussusception in the vascular reconstruction (A). Kidney recovery during Thy1.1 nephritis is associated with proliferation of glomerular cells (B), increased glomerular volume $(\mathbf{C})$, and reduced total number of glomeruli (D). Columns were compared versus healthy animals (ctrl) (A, C and D: $n=4 ; \mathbf{B}: n=4$ in ctrl, day 5 , day 14 , and day 21 groups, $n=5$ in day 3 group, and $n=8$ in day 9 group). ${ }^{*} P<0.05,{ }^{* * *} P<0.01,{ }^{* * * *} P<0.0001$. 


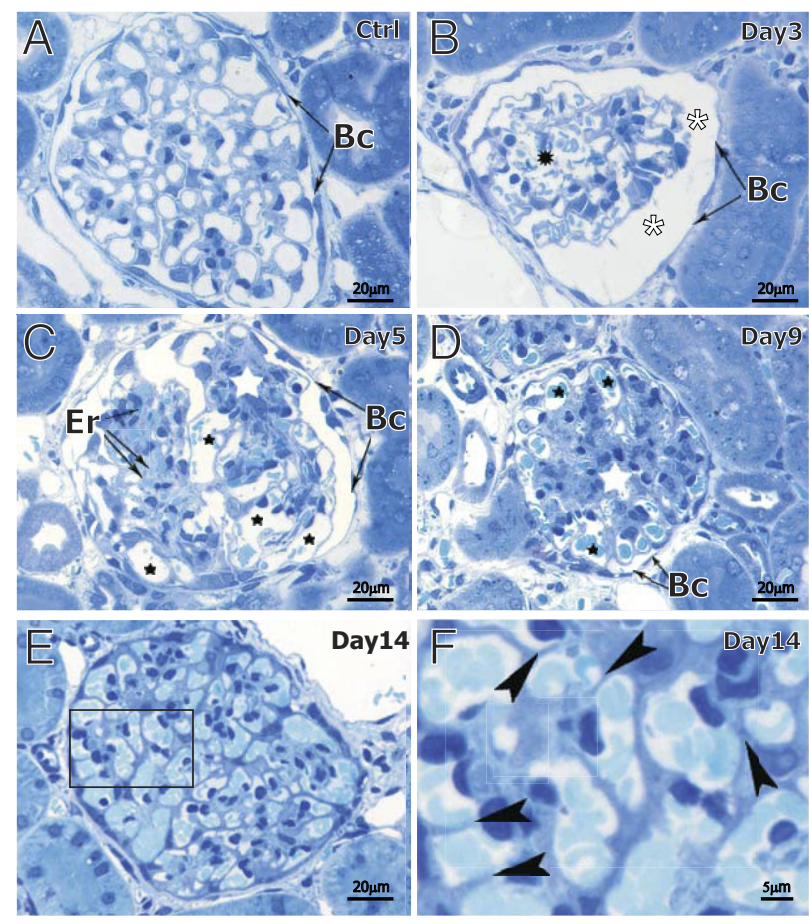

Figure 3. Toluidine blue staining of semithin rat kidney sections at different stages of Thy1.1 nephritis. Capillaries of healthy glomeruli (A) assemble in a compact structure with inconspicuous mesangial region. Day 3 of nephritis (B) is characterized by the presence of first microaneurysms (black asterisk) and wide urinary space (white asterisks). At day $5(\mathbf{C})$, enlarged capillaries (black asterisks) and microaneurysms are frequently filled with erythrocytes $(\mathrm{Er})$. Starting from day $5(\mathbf{C})$, but very pronounced by day $9(\mathbf{D})$, is the prominent, dense mesangial region (white asterisks), which pushed the dilated capillaries to the periphery of glomerulus (black asterisks). At day 14 (E and $\mathbf{F}$ ), intussusception (ie, pillar formation) is represented here as an insertion of tiny walls between the capillaries (arrowheads in $\mathbf{F}$, which is a zoom of the area surrounded by the rectangle in $\mathbf{E}$ ). $\mathrm{Bc}=$ Bauman capsule.

walls between the capillaries (Figure 3, E and F; rectangle and arrowheads). This observation is in line with the onset of intussusceptive vessel splitting.

\section{Glomerular Immunoreactivity of VEGFR2/VEGF Increases during Thy1.1 Nephritis}

Immunohistochemical staining of VEGFR2NEGF was used to determine their in situ expression pattern in healthy and nephritic glomeruli. The intensity of both VEGFR2 and VEGF immunoreactivity increased during the nephritis course compared with healthy glomeruli (Figure 4, A-J). The expression pattern of VEGFR2/NEGF during nephritis was confirmed by Western blot analysis (Figure 4K). The upregulation lasted until day 14 of nephritis, whereas at day 21 VEGFR2NEGF expressions have already decreased and were comparable with basal levels (Figure 4K). The upregulation of VEGFR2NEGF resulted in an increase of receptor phosphorylation (Figure 4L).

Next, we performed a colocalization study to find out what types of cells express VEGFR2 in healthy and nephritic glomeruli. In both cases we observed a marked degree of colocalization between VEGFR2 and CD31, an endothelial cell marker. In nephritic glomeruli, colocalization was observed at the periphery of lobules (Figure $4 \mathrm{~N}$ ), whereas in control animals, costaining was detected throughout the entire glomerulus (Figure 4M). We also noticed that the intensity of glomerular VEGFR2 staining was lower overall compared with the intensity of tubular VEGFR2 labeling. Endothelial cells were not the only glomerular cells expressing VEGFR2 during nephritis. As Figure 40 shows, we could detect colocalization between $\alpha$-SMA and VEGFR2 in the central part of glomerular lobules, indicating that injured mesangial cells express VEGFR2.

\section{Administration of PTK/ZK during Nephritis Results in the Reduction of $\alpha$-SMA and Tensin Expression and Transient Decrease of Glomerular Volume}

A total of $50 \mathrm{mg} / \mathrm{kg}$ of PTK/ZK administered i.p. inhibited glomerular VEGFR2 signaling by blocking receptor overexpression observed during Thy1.1 nephritis. Thus, the amount of phosphorylated VEGFR2 decreased (Figure 5, $A$ and $B$ ). Expression and phosphorylation of PKB, a molecule involved in VEGFR2 signaling, ${ }^{19}$ were lower in glomeruli of nephritic PTK/ZK-treated animals compared with glomeruli isolated from vehicle-treated nephritic animals (Figure 5, A and C). PDGFR $\beta$ receptor was overexpressed in the nephritic glomeruli at days 9 and 14 of nephritis, and PTK/ZK compound blocked the expression of phosphorylated receptor similarly to VERGFR2 (Figure 5, D and E). In contrast to VEGFR2 and PDGFR $\beta$ receptors, glomerular VEGFR1 expression showed only a mild but not statistically significant regulation that was not influenced by PTK/ZK treatment (Figure 5, D and F).

Next, we aimed to investigate whether VEGFR2 inhibition had an effect on nephritis-induced glomerular hypertrophy. Glomerular volume was quantified from vascular casts by means of SEM. PTK/ZK significantly decreased glomerular volume when applied for 5 and 9 days. At days 14 and 21 of nephritis, glomerular volume was again comparable to the vehicle-treated nephritic animals (Figure 5G).

To determine whether PTK/ZK influenced mesangial cell behavior, we analyzed glomerular expression of $\alpha$-SMA, tensin, Thy1.1, fibronectin, and collagen IV proteins. As the Western blot analysis shows, $\alpha$-SMA was not detected in control glomeruli but was significantly expressed at day 5 of nephritis. Surprisingly, PTK/ZK treatment led to a decrease of $\alpha$-SMA expression (Figure 5, $\mathrm{H}$ and I). Tensin, a protein also described to be specifically expressed by mesangial cells, was overexpressed in nephritic glomeruli and downregulated by PTK/ZK (Figure 5, $\mathrm{H}$ and J). Thy 1.1 molecule was abundant in healthy glomeruli, but its level decreased on nephritis induction due to the mesangiolysis. PTK/ZK treatment did not influence the expression of Thy1.1 during nephritis (Figure 5, $\mathrm{H}$ and $\mathrm{K}$ ). Expression of fibronectin and collagen type IV increased in the glomeruli of nephritic rats, and their levels were not regulated by PTK/ZK (Figure 5, H, L, and M).

\section{PTK/ZK Administration Leads to Glomerular Hemorrhaging}

Morphologic evaluation by semithin sections revealed structural changes in the nephritic nephrons on PTK/ZK 


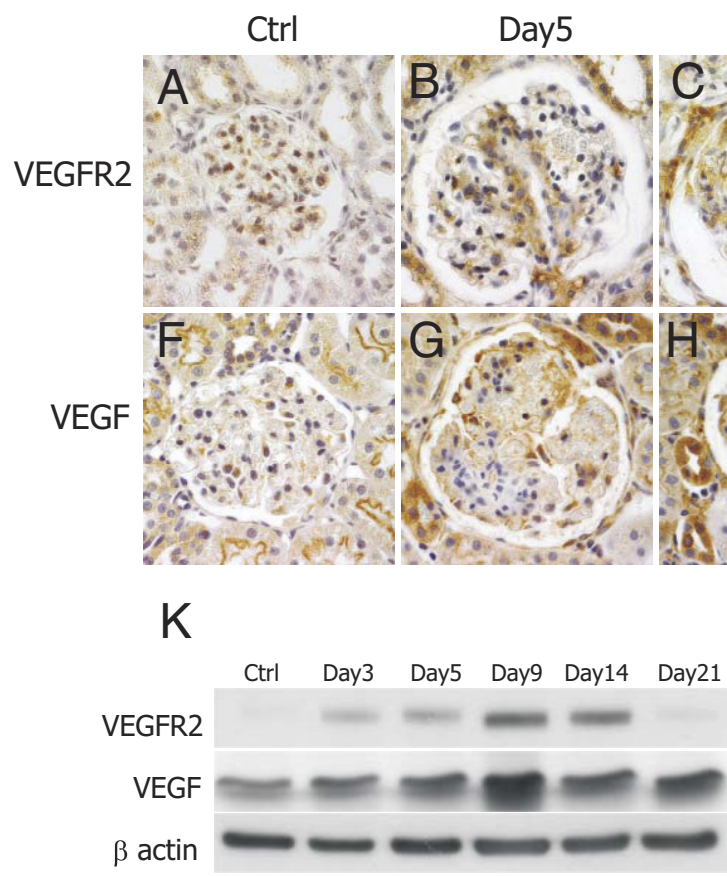

Day9 Day14

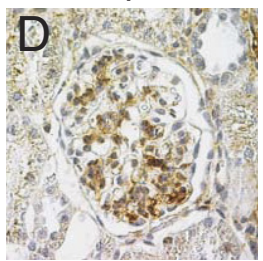

dit $=2$ a 9 :
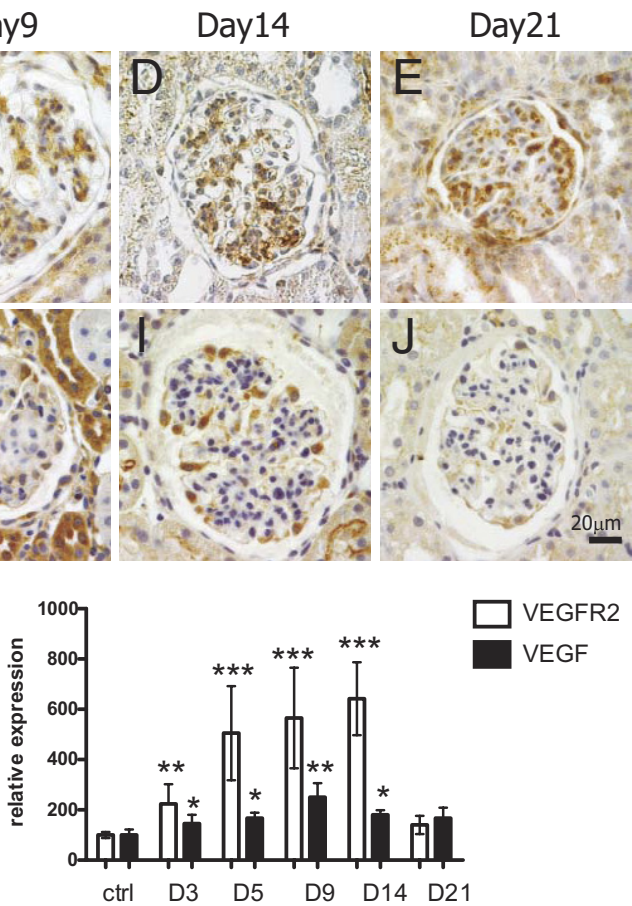

VEGFR2

VEGF

day of nephritis

$\mathrm{L}$
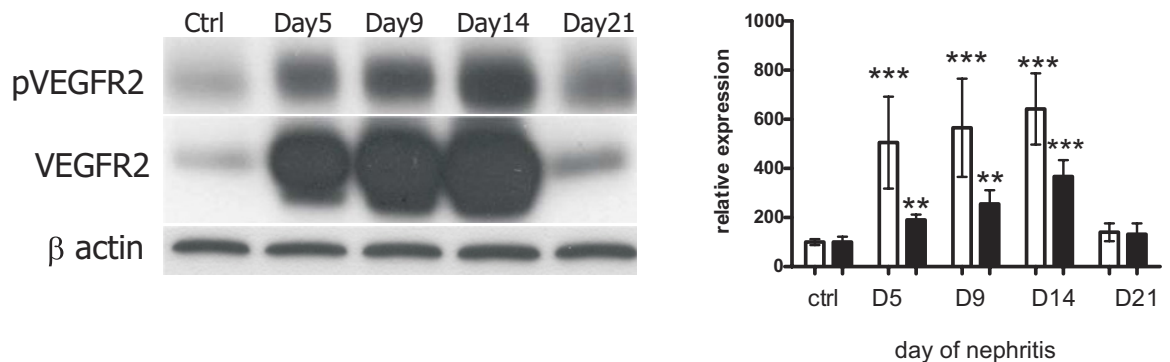

VEGFR2

pVEGFR2

\section{CD31/VEGFR2}

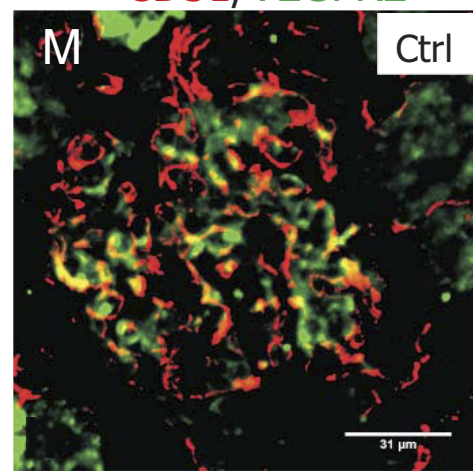

CD31/VEGFR2

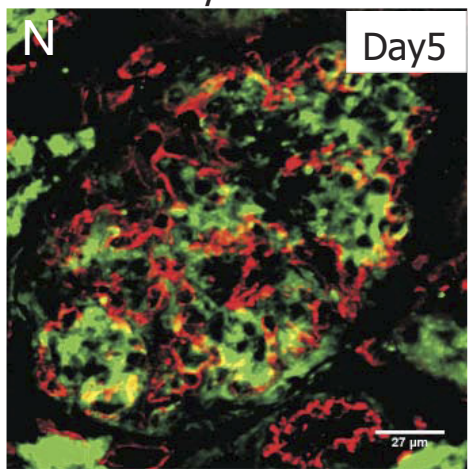

aSMA/VEGFR2/DAPI

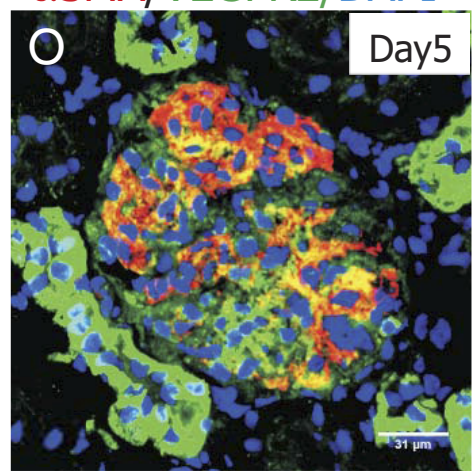

Figure 4. Analysis of glomerular VEGFR2/VEGF expression and signaling during Thy1.1 nephritis. Immunohistochemical staining of VEGFR2 (A-E) and VEGF (F-J) showed that both receptor and ligand were expressed at low levels in healthy glomeruli but were up-regulated during the nephritis course. Western blot analysis of glomerular VEGFR2/VEGF expression confirmed receptor and ligand up-regulation after OX7 injection (K, $n=4)$. Extent of VEGFR2 phosphorylation increased concomitantly to the increase of VEGFR2 expression $(\mathbf{L}, n=4)$. Glomerular VEGFR2 colocalized with CD31 in healthy $(\mathbf{M})$ and nephritic $(\mathbf{N})$ glomeruli. At day 5 of nephritis, colocalization between VEGFR2 and $\alpha$-SMA could be additionally detected $(\mathbf{O}) .{ }^{*} P<0.05,{ }^{* * *} P<0.01,{ }^{*}{ }^{* * *} P<0.0001$.

treatment. The PTK/ZK-treated group was characterized by the presence of intraglomerular hemorrhages. The hemorrhagic areas were located in the mesangium, but they often broke into the urinary space and into the tubular system (Figure 6, A, B, and C). The presence of hemorrhages in the glomeruli and tubuli was confirmed by SEM on kidneys dried at critical point (Figure 6, D and E). This fact may play a crucial role in 

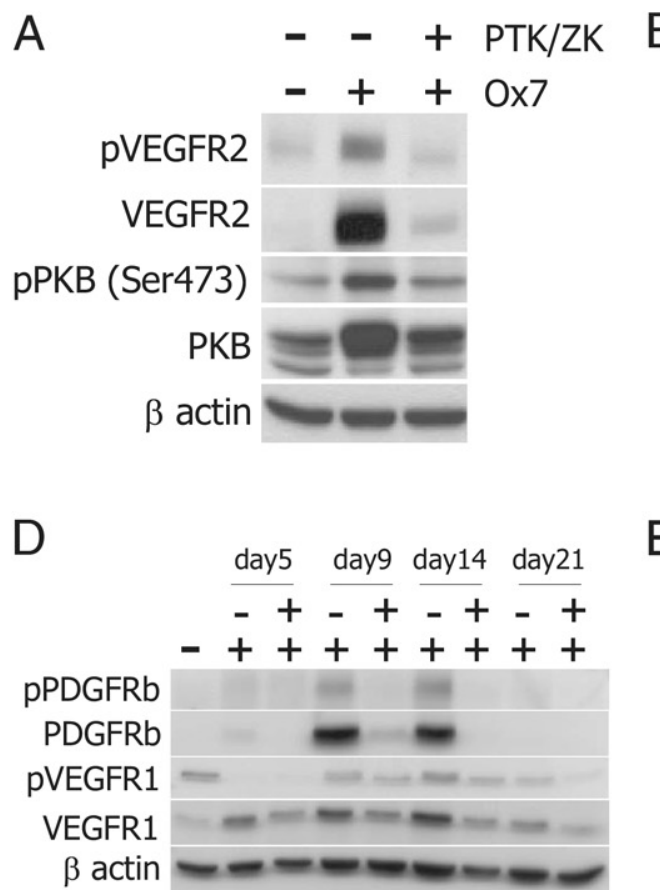

G

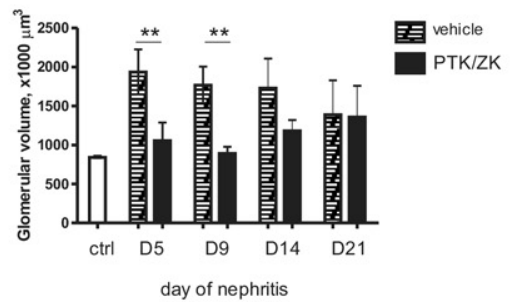

$\mathrm{K}$

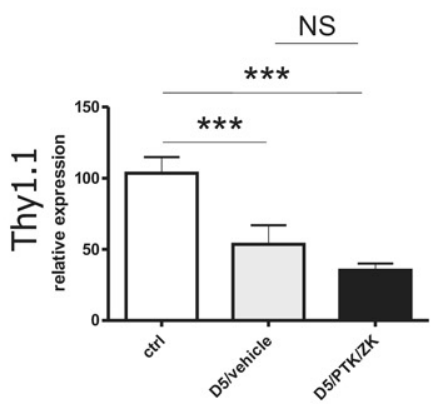

B

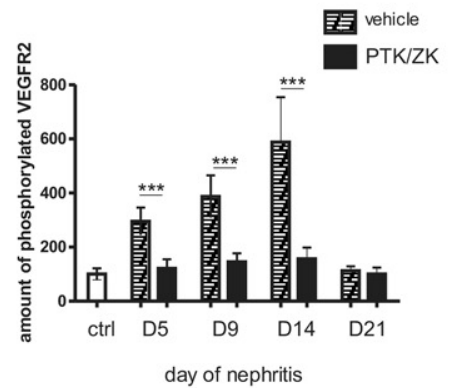

$E$

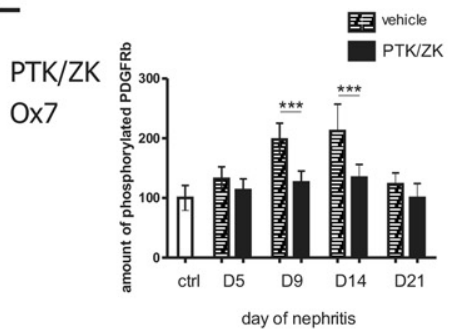

F
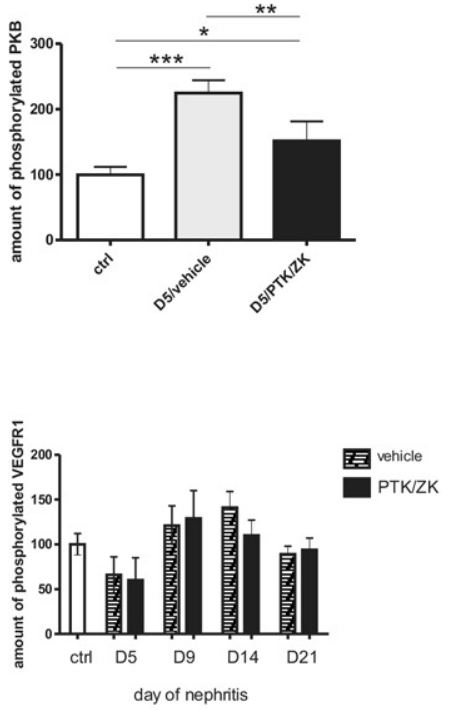

$\mathrm{H}$

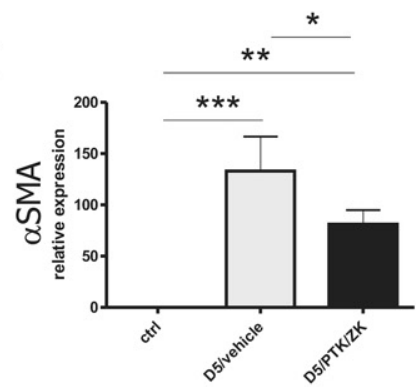

Thy1.1

$\beta$ actin

fibronectin

collagen IV

$\beta$ actin

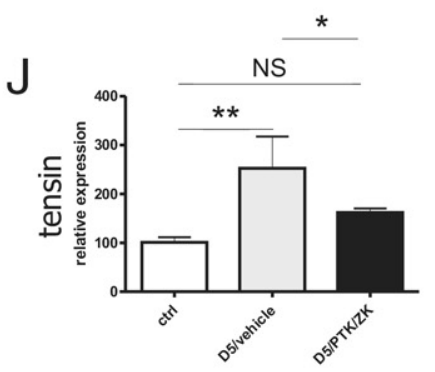

L

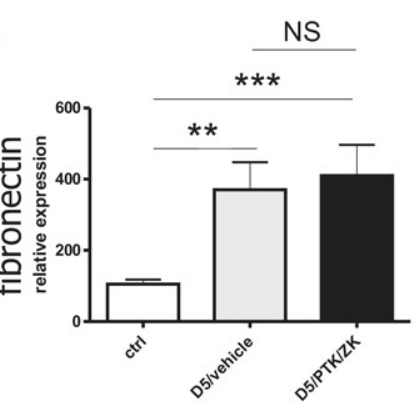

M

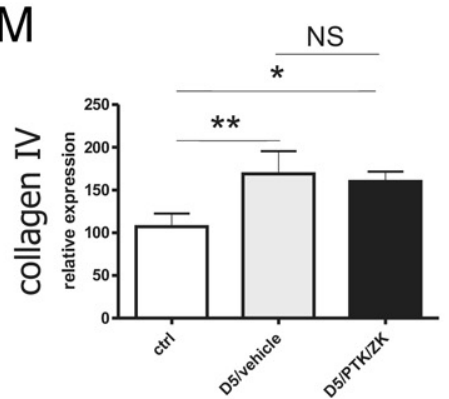

Figure 5. Effect of PTK/ZK on receptor tyrosine kinase signaling, glomerular volume, and mesangial cells during nephritis. PTK/ZK administered i.p. during nephritis led to the inhibition of glomerular expression and phosphorylation of VEGFR2 (A and $\mathbf{B}, n=4)$ and PDGFR $\beta$ (D and E, $n=4)$ but not VEGFR1 (D and $\mathbf{F}, n=4)$. Glomerular expression and activity of PKB was partially inhibited as a result of PTK/ZK treatment $(\mathbf{A}$ and $\mathbf{C}, n=4)$. PTK/ZK transiently prevented nephritis-induced glomerular hypertrophy $(\mathbf{G}, n=3-4)$. Treatment with PTK/ZK resulted in the decrease of $\alpha$-SMA and tensin expression, but the compound did not influence the expression of Thy1.1, fibronectin, and collagen type IV (H). Expression of these proteins was normalized to $\beta$-actin and quantified with Image software (I-M, $n=4) .{ }^{*} P<0.05,{ }^{* *} P<0.01,{ }^{* * * * *} P<0.0001$. 

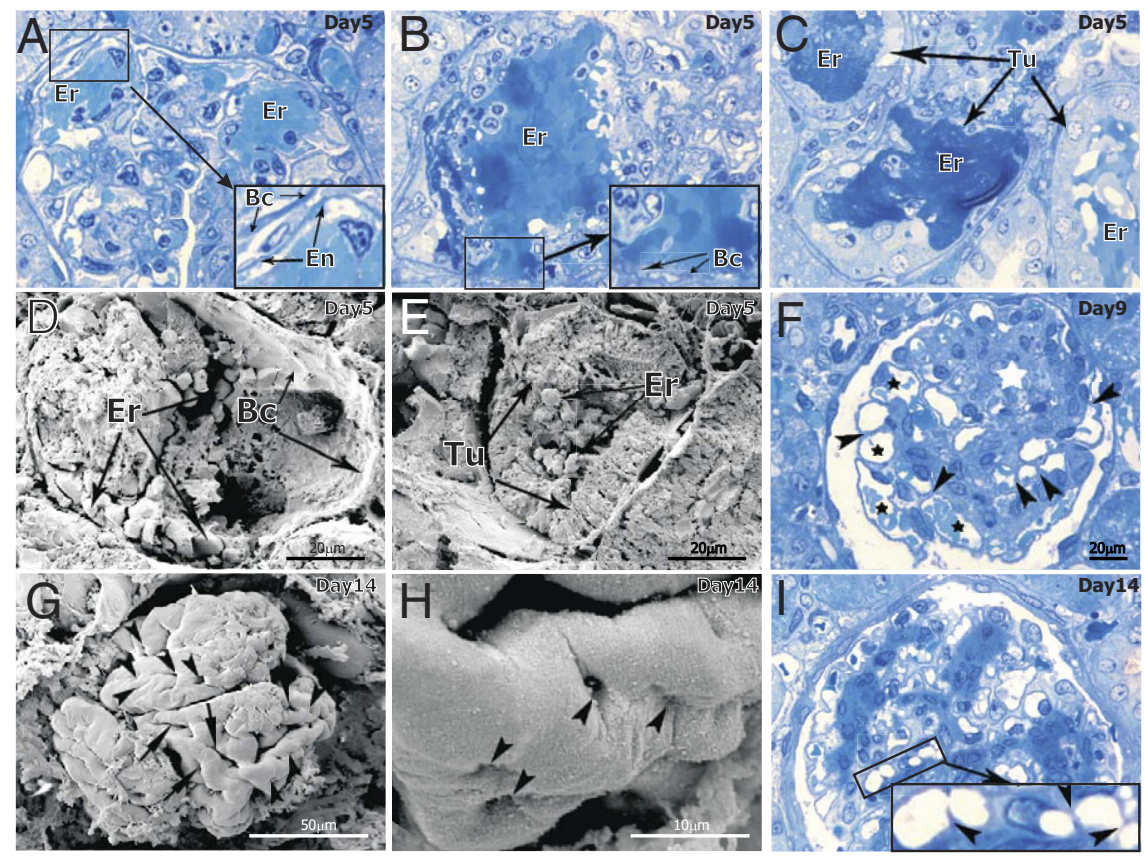

Figure 6. Glomerular histopathologic alterations after PTK/ZK treatment of nephritic rats. Dilated vessels and microaneurysms with intact vascular endothelial walls were observed at the periphery of the glomeruli (A; see corresponding zoom), but most often the breach of the glomerular vessel wal (B) led to the hemorrhages into the urinary space with penetration of blood into the tubular system (C). SEM on kidneys dried at critical point confirmed the presence of intraglomerular (D) and tubular (E) hemorrhages at day 5 of nephritis. After 9 days of PTK/ZK treatment $(\mathbf{F})$, enlarged area of mesangial cells (white asterisk) with ongoing pillar formation (arrowheads) in the dilated capillaries (black asterisks) was observed. Using SEM we could detect a high number of solitary pillars ( $\mathbf{G}$ and $\mathbf{H}$, arrowheads) merging and forming capillary loops and slits (G, arrows). The semithin method showed that at this time point (I) capillaries became smaller in diameter, are better seen in the medullar part of glomeruli, and show signs of intussusceptive angiogenesis (zoom in $\mathbf{I}$ ). En = endothelium; Bc = Bauman capsule; $\mathrm{Er}=$ erythrocytes; $\mathrm{Tu}=$ tubuli. A, B, C, F, and I: Stained with toluidine blue.

the subsequent degeneration of nephrons, starting with glomeruli and expanding to the tubular system. It can also explain the reduced number of microaneurysms on vascular casts in the PTK/ZK-treated group (see Supplemental Figure S1B at http://ajp.amjpathol. org). At day 9 of nephritis, hemorrhages were in the process of organization and the mesangial area was still enlarged (Figure 6F, white asterisk). We could still observe the invasion of capillary lumen by the thin capillary walls, an evidence of intussusception (Figure 6, F and I; arrowheads). The presence of tiny holes (Figure 6, $\mathrm{G}$ and $\mathrm{H}$; arrowheads), small capillary loops, and slits (Figure 6G, arrows) during PTK/ZK treatment was confirmed by SEM on kidneys dried at critical point.

\section{PTKIZK Administration Impairs Filtration in Nephritic Glomeruli}

Next, we aimed to examine whether PTK/ZK administration impairs glomerular permeability in nephritic rats. Fluorescent spheres (diameter, $100 \mathrm{~nm}$ ) were injected intravenously, and rats were sacrificed 30 minutes later. To determine whether the spheres are located in the Bowman space or within glomerular vasculature or mesangium, the cryosections were additionally stained with synaptopodin. We could see mild focal fluorescence in the glomeruli of healthy rats (Figure 7A). This signal disappeared in the glomeruli of nephritic vehicle-treated animals (Figure 7B). However, after PTK/ZK treatment, nephritic glomeruli showed very bright red fluorescence (Figure 7, C-E). The spheres were mainly located in mesangial cells (Figure 7D) and ED1-positive macrophages (Figure 7E).

\section{The Vasculature of PTK/ZK-Treated Nephritic Animals Shows Multiple Abnormalities But Intussusception Is Affected Only After Long- Term Treatment}

The most striking effects of PTK/ZK on glomerular vessel structure were observed with vascular casting and SEM. At day 5 of nephritis, glomerular capillaries appear to be tightly connected (Figure 8A), and the number of microaneurysms was significantly smaller in PTK/TK-treated group (see Supplemental Figure S1B at http://ajp.amjpathol. org). Higher magnification revealed the presence of tiny holes and small capillary loops (Figure 8B, arrowheads
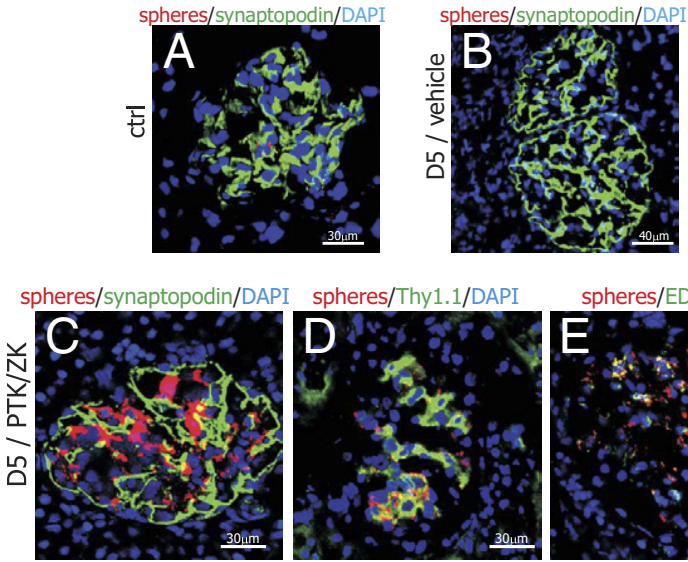

spheres/ED1/DAPI

Figure 7. Study of glomerular filtration by the injection of fluorescent microspheres. Red fluorescent spheres of a diameter of $100 \mathrm{~nm}$ were observed only sporadically in the glomeruli of healthy rats (A) and were not present in nephritic glomeruli (B) when intravenously injected to animals. When nephritic rats were treated with $\mathrm{PTK} / \mathrm{ZK}$ for 5 days, the fluorescence of the spheres was clearly visible in the glomerular area $(\mathbf{C})$. Spheres were taken up by the Thy1.1positive mesangial cells (D) and ED1-positive macrophages (E). 


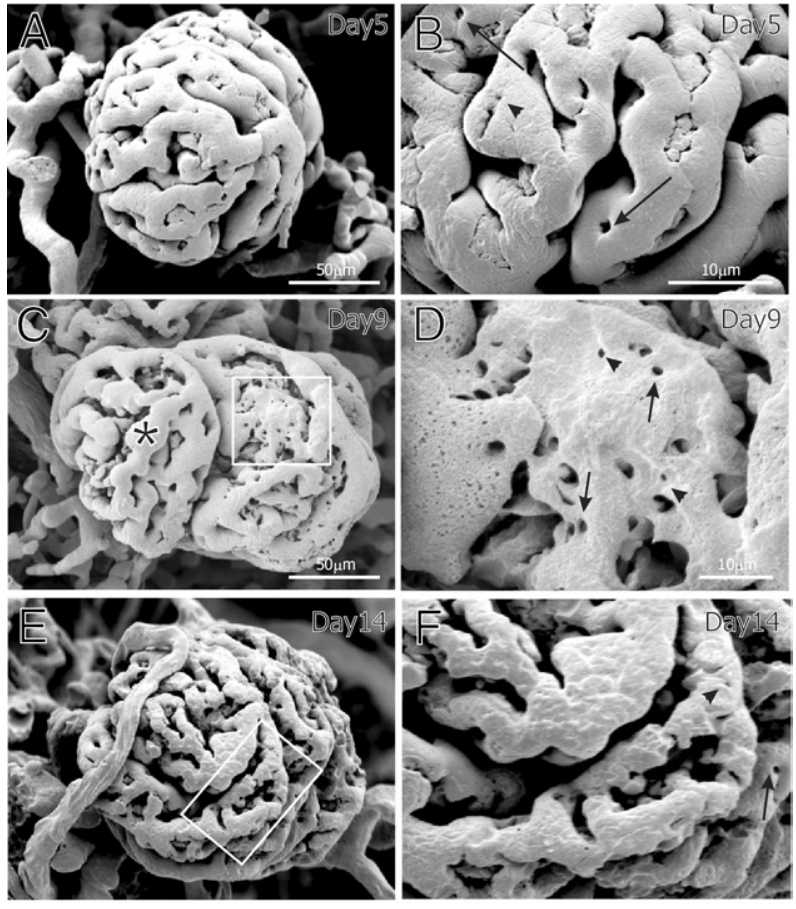

G density of tiny holes

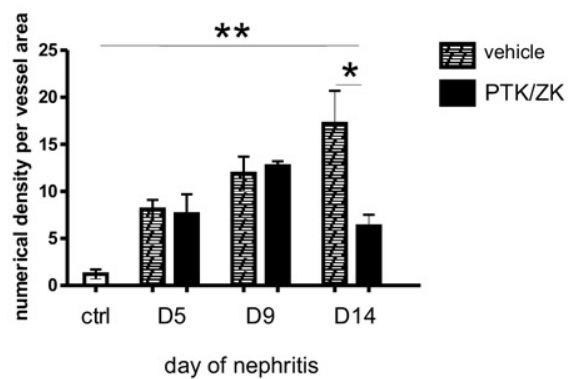

$\mathrm{H}$

density of small capillary loops

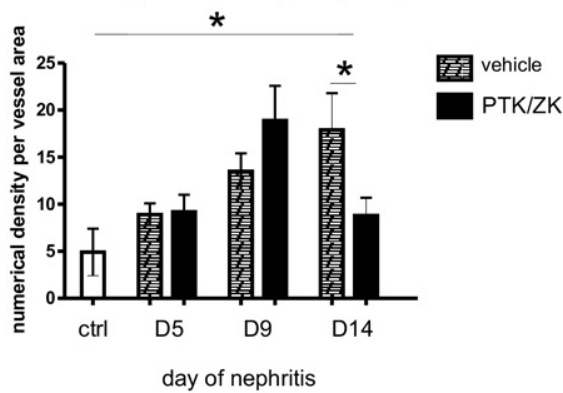

Figure 8. Vascular cast and SEM of nephritic glomerular vasculature after PTK/ZK treatment. At day 5 of nephritis, glomerular capillaries appeared to be tightly connected (A); higher magnification revealed ongoing intussusception (B). Glomerulus at day 9 of nephritis is depicted in $\mathbf{C}$. One lobule is characterized by haphazardous pillar formation process (D). The capillaries of the second lobule are tortuous $(\mathbf{C}$, asterisk). Glomerular vasculature at day 14 of nephritis $(\mathbf{E}$ and $\mathbf{F})$ was characterized by uneven endothelial cell surface, tortuous capillary edges, and presence of tiny holes and small capillary loops. $\mathbf{B}, \mathbf{D}$, and F: Tiny holes are marked with arrowheads and small capillary loops with arrows. $\mathbf{D}$ and $\mathbf{F}$ : Magnified areas depicted by rectangles on $\mathbf{C}$ and $\mathbf{E}$, respectively. Numerical density of pillars $(\mathbf{G})$ and small loops $(\mathbf{H})$ was reduced only after 14 days of PTK/ZK treatment compared with vehicle-treated animals nephritic at respective time points. The number of tiny holes and small capillary loops in PTK/ZK-treated animals was, however, up-regulated compared with healthy animals (ctrl) $(n=4) .{ }^{*} P<0.05,{ }^{* * * *} P<0.01$. and arrows, respectively). The vessel structure differed, however, from what was observed in nephritic vehicletreated glomeruli (Figure 1). The surface of glomerular endothelial cells appears not to be homogenous and tortuous (Figure 8B). Figures $8, \mathrm{C}$ and D, present the glomerulus of a PTK/ZK-treated rat at day 9 of nephritis. One lobule shows pillar formation process (Figure 8, C and magnified area at D), although pillars arise haphazardly. Capillaries of the second lobule (Figure 8C, asterisk) are abnormally shaped without clear contour. Those features were more pronounced the longer PTK/ZK was administered. At day 14 we could see that the endothelial cell surface is fiberlike, vessel edges are tortuous, and the capillaries appear irregular (Figure 8, E and magnified area at F). We detected tiny holes and small capillary loops only rarely in comparison with Thy. 1 nephritic glomeruli (Figure 8F, arrowheads and arrows, respectively). The morphometric analysis of intussusception onset showed that the appearance of tiny holes or small capillary loops in PTK/ZK-treated rats did not differ from vehicle-treated nephritic animals at days 5 and 9 . Although intussusception decreased after 14 days of PTK/ZK administration compared with vehicle-treated nephritis animals, the number of tiny holes and small capillary loops in PTK/ZK-treated group was still significantly up-regulated compared with healthy animals (Figure $8, \mathrm{G}$ and $\mathrm{H}$ ). This indicates that PTK/ZK administration did not completely block intussusception onset at this time point.

Although different morphologic evaluations showed that glomeruli structure was affected by PTK/ZK treatment, we failed, however, to observe any effect of PTK/ZK on urinary protein excretion (see Supplemental Figure S2 at $h$ ttp://ajp.amjpathol.org).

\section{Discussion}

The present work uses different methods to follow the revascularization process and focus on pathways leading to glomerular recovery during Thy 1.1 nephritis and their molecular basis (Figure 9). We used different methods to follow the revascularization process that occurred by intussusceptive angiogenesis. We next asked the question of the involvement of combined VEGFR and PDGFR $\beta$ inhibition in the different aspects of recovery process.

\section{Intussusceptive Angiogenesis Restores Glomerular Capillaries and Kidney-Specific Angioarchitecture}

The restoration of capillary structure can theoretically occur via sprouting or intussusceptive angiogenesis or a combination of both. ${ }^{14,20}$ Intussusception is a newly characterized angiogenic mode, and it has recently been shown to take place during glomeruli recovery from injury. ${ }^{11}$ Its characteristic features, namely, low endothelial proliferation, short duration, and physiologic levels of transpermeability, make this process suitable for regenerative purposes. ${ }^{3-5}$ Herein, we confirm that a robust intussusception onset takes place during Thy 1.1 recovery. We did not observe any sprouts arising from glomer- 


\section{Day3}
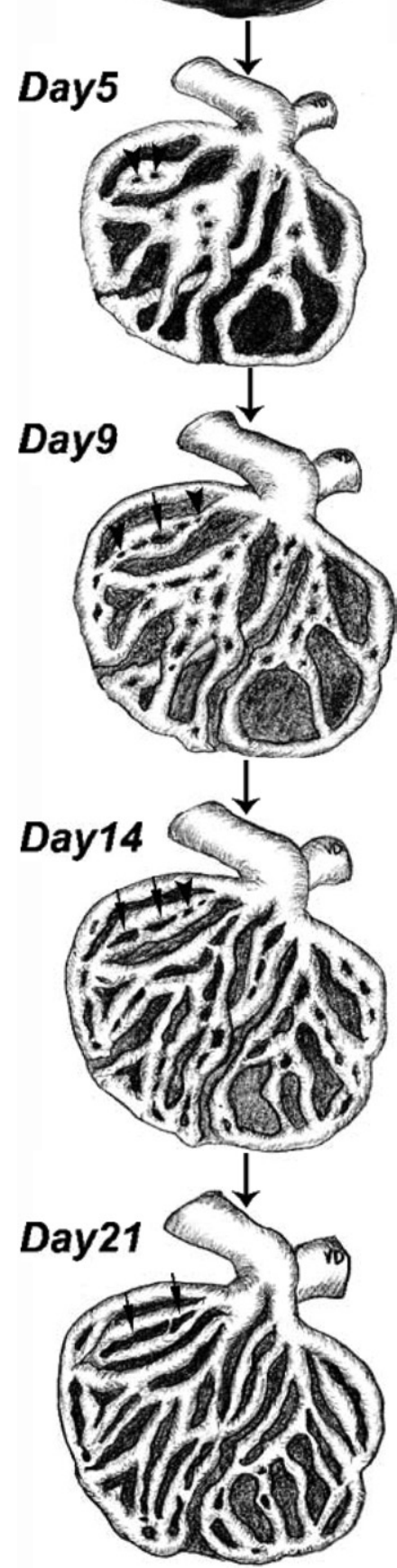

Figure 9. A schema showing the restoration of capillary structure and kidney-specific angioarchitecture after induction of Thy 1.1 nephritis. By day 5 , many pillars (arrowheads) arise randomly in the capillary plexus. From day 9 to day 21 pillars reshape, elongate, and fuse, forming small capillary loops (arrows) and thus splitting vascular segments and resulting in the formation of slender, solitary capillary entities. The latter events restore the classical glomerular angioarchitecture. ular vessels on Thy1.1 nephritis induction. Quantification of tiny holes and small capillary loop density showed that intussusception peaks at day 14 of nephritis. Indeed, at this time point endothelial cell number has already recovered and little cell proliferation was observed. The peak of intussusception is preceded by dilatation of capillaries, even by microaneurysm formation. This phenomenon has previously been described during avian kidney and lung development ${ }^{6,7}$ and recently during chorioallantoic membrane enlargement (data not published). Intussusception should be associated with capillary dilation before the insertion of the transluminal pillars and vessel splitting. In our study, however, the capillaries were more compact, and almost no microaneurysms were detected when nephritic animals were treated with PTK/ZK. We therefore speculate that glomerular hypertrophy is not a prerequisite for the insertion of a pillar to the vessel lumen. Interestingly, we sporadically observed the presence of tiny holes on the surface of microaneurysms. At present, it is not known if and how microaneurysms heal, but it is possible that the transluminal pillar formation (ie, intussusceptive process) splits and reorganizes the expanded vascular volume, at least in some microaneurysms. The new vascular segments created this way are subsequently requited into the microvascular circulation.

We additionally show that although Thy 1.1 nephritis leads ultimately to healing, the number of nephrons is reduced by $39 \% 3$ weeks after nephritis induction. At the same time, the remaining glomeruli are on average $65 \%$ larger. Intussusceptive angiogenesis contributes to capillary regrowth in the single glomerulus; it is therefore conceivable that this angiogenic process restores the entire kidney filtration surface by increasing capillary volume of glomeruli that survived the injury. The same mechanism probably takes place in the kidneys of uninephrectomized rats. ${ }^{21}$ We hypothesize that the increase of glomerular volume is a compensatory mechanism for the loss of nephrons during nephritis.

\section{The Effects of PTKIZK on VEGFR1, VEGFR2, and PDGFR $\beta$ Expression and Phosphorylation in Nephritic Glomeruli and Their Implications for Nephritis Resolution}

VEGFR/VEGF and PDGFR $\beta / P D G F B$ are well-described pairs of receptor tyrosine kinases and their ligands that play a major role in angiogenesis. ${ }^{15}$ Recent progress of understanding renal diseases showed that many of them arise from inadequate angiogenic response on injury and that manipulation of these signaling pathways may influence disease outcome. ${ }^{22,23}$

The data concerning VEGFR/VEGF importance for nephritis recovery are plentiful but contradictory; however, the outcomes are thought to dependent on the nephritis type and thus on underlying pathophysiology. ${ }^{24-28}$ In the particular case of Thy 1.1 nephritis, VEGF blocking had an adverse effect on endothelial cell recovery. ${ }^{24}$ On the other hand, up-regulation of PDGFR $\beta$ in the injured mesangial cells was shown to be an adverse phenomenon because many studies reported an improvement of glo- 
merular recovery from Thy 1.1 nephritis after PDGFR $\beta /$ PDGFB blockade. ${ }^{29,30}$ Interestingly, PDGFC, a ligand for PDGFR $\alpha$, was shown recently to be an important factor for glomerular endothelial repair. ${ }^{31}$ The effects of PDGFR family on glomerular recovery from nephritis are therefore specific for particular receptor and its ligand.

Our results show that PTK/ZK blocks not only VEGFR2 and PDGFR $\beta$ activation but also expression in vivo. The phenomenon of down-regulation of VEGFR2 expression by the small-molecular-weight inhibitor was already reported by others, ${ }^{32,33}$ but the mechanism is lacking. We have not observed a statistical significance in the glomerular VEGFR2 mRNA expression between vehicle and PTK/ZK-treated rats, although the tendency toward lower levels in PTK/ZK-treated group was clearly visible (data not shown). It is additionally interesting that PTK/ZK did not inhibit VEGFR1, although the affinity of the compound is higher for VEGFR1 than for PDGFR $\beta .{ }^{13}$ However, VEGFR1 is mildly regulated during Thy 1.1 nephritis compared with VEGFR2 and PDGFR $\beta$. We therefore hypothesize that the mechanism by which PTK/ZK down-regulated the expression of the underlying receptor is dependent not only on the phosphorylation status of the receptor but also on pathways that allow the receptor to be overexpressed at the sites of active angiogenic process. Further studies are, however, needed to sufficiently explain this phenomenon.

We further show that phosphorylation of PKB, a kinase downstream of VEGFR2, is diminished on PTK/ZK administration. The level of PKB inhibition is not complete because this intracellular signaling molecule is regulated by a variety of upstream factors. ${ }^{34}$

\section{Possible Implications of Mesangial $\alpha$-SMA and Tensin Down-Regulation by PTK/ZK: Presence of Intraglomerular Hemorrhages, Reduced Number of Microaneurysms, and Glomerular Volume}

PTK/ZK administration resulted in the presence of intraglomerular hemorrhages and a decreased number of microaneurysms at day 5 of nephritis. We think that this phenomenon is related to the VEGFR2 inhibition rather than PDGFR $\beta$ inhibition because at day 5 of nephritis, PDGFR $\beta$, in contrast to VEGFR2, is not yet up-regulated and thus PTK/ZK did not inhibit this signaling. It is however conceivable that at later time points, inhibition of PDGFR $\beta$ in addition to VEGFR2 has a major contribution to the effects of PTK/ZK on glomerular recovery from Thy 1.1 nephritis. Injured mesangial cells express de novo $\alpha$-SMA, which is a commonly used marker of mesangial cell activation and proliferation. ${ }^{35}$ On the other hand, Thy 1.1 molecule is thought to be constitutively expressed by mesangial cells. ${ }^{12,36,37}$ Our data show that although glomerular $\alpha$-SMA expression was decreased in the PTK/ ZK-treated animals, the compound had no effect on Thy1.1 molecule expression. Thus, PTK/ZK seems not to influence mesangial cell number (ie, mesangiolysis occurs), but injured mesangial cells do not express $\alpha$-SMA.
Treatment with PTK/ZK down-regulated not only $\alpha$-SMA but also tensin expression. The latter protein was shown to be expressed at the mesangial protrusions, ${ }^{38}$ similarly to $\alpha$-SMA. ${ }^{12}$ Tensin is hypothesized to be an important molecule for the contractile apparatus of mesangial cells. ${ }^{38}$ Overexpression of $\alpha$-SMA and tensin during nephritis could therefore allow mesangial cells to better support vessels that recover from injury, and when this phenotypic change is prevented by PTK/ZK, mesangial cells lose their specific features and let endothelium be fully vulnerable to antiangiogenic therapy. Indeed, the vascular casting method, which is in fact the cast of endothelial cells covering the lumen of perfused vessels, showed that glomerular capillary surface is malformed and tortuous. We hypothesize that the walls of sinusoidal and microaneurysm-like vessels remain poorly covered by mesangial cells and thus become fragile. Capillaries rupture and result in the occurrence of multiple hemorrhages often detectable in the tubular system and accounting for the nephron degeneration. As a result, glomerular volume remains reduced and fewer microaneurysms can be detected.

\section{Impaired Glomerular Filtration After PTK/ZK Administration}

To examine the impact of PTK/ZK on glomerular filtration, we used fluorescent spheres of a 100-nm diameter, which is approximately the size of fenestrations in healthy glomeruli. ${ }^{39}$ During nephritis, glomerular capillaries are destroyed and thus the permeability is higher. Therefore, the spheres could go through the glomerular sieve within 30 minutes and no red fluorescence could be observed. Serendipitously, PTK/ZK led to the accumulation of spheres in the glomerular mesangial cells and macrophages. This could be explained by the fact that antiVEGF therapy leads to the loss of glomerular fenestrations $^{33}$ and the filtration of spheres is blocked. Under those circumstances, spheres are taken up by macrophages. As mesangial cells and generally pericytes are implicated to have phagocytic, macrophage-like properties, ${ }^{40}$ they could contribute to the "cleaning process."

\section{Kidney Regeneration Under PTK/ZK Treatment as a Part of Anticancer Therapy}

It is generally accepted that angiogenesis in tumors is crucial for the growth and dissemination of neoplasm, which is the rationale for the application of angiogenesis inhibitors as anticancer therapy.

At the time there are more than 1800 clinical trials

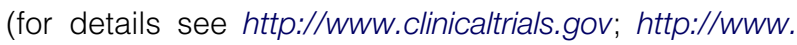
nci.nih.gov/clinicaltrials) dealing with antiangiogenic compounds, mostly inhibitors of VEGF. PTK/ZK was also recently evaluated in the phase 1 clinical trial for the treatment of solid cancers. ${ }^{41}$ Despite the partial regression of tumor vessels and reduction in tumor size, the effects are transient and long-term results are disappointing. A recently published article by Hlushchuk et $\mathrm{al}^{14}$ reported that a switch from sprouting to intussusceptive angiogen- 
esis might represent an adaptive response to treatment with PTK/ZK. At the same time intussusceptive angiogenesis seems to be essential during kidney development and recovery after Thy 1.1 nephritis. ${ }^{5,6,11}$ Taken together and in the light of the data presented in this article, we conclude that most likely an antiangiogenic therapy with PTK/ZK compound does not influence significantly the kidney recovery and glomerular restoration after acute Thy 1.1 nephritis. This is probably a net effect of inhibiting two pathways, namely, VEGFR2 and PDGFR $\beta$, with opposite roles in the recovery from acute mesangioproliferative glomerulonephritis. However, it is possible that PTK/ZK may have more pronounced long-term effects on kidney function. Furthermore, we cannot exclude that PTK/ZK has additional effects we are at the moment not aware of; for example, it was recently shown that PTK/ZK inhibits aromatase activity in breast cancer cell line. ${ }^{42}$ These facts could have important clinical implication for patients with nephritis who are receiving antiangiogenic treatment.

\section{PTK/ZK Administration Affects Intussusceptive Angiogenesis Only After Long-term Treatment}

Although PTK/ZK prevented glomerular hypertrophy at days 5 and 9 of nephritis, it did not significantly change the dynamics of intussusception onset. It is however interesting that intussusception was inhibited after 14 days of treatment in contrast to shorter time points. Perhaps this is because different factors and cofactors regulate the pillar formation process and, as the resolution of nephritis progresses, they are differently expressed and thus differently affected by PTK/ZK treatment. Because PDGFR $\beta$ signaling is of major importance for pericyte functions and this receptor is expressed most prominently on day 14 of nephritis, it is conceivable that PDGFR $\beta$ is at least one of these factors. However, the fact that intussusception still takes place despite PTK/ZK administration is in line with the observation that intussusception, but not sprouting, is the main angiogenic mode in tumors treated with PTK/ZK. ${ }^{14}$

\section{Acknowledgments}

We acknowledge technical support from Brigitte Scolari, Regula Buergy, and Clemens Weber. We thank Andrew Makanya for the critical reading of the manuscript, Dean Evans (Novartis Pharma AG, Basel, Switzerland) for providing us the compound PTK/ZK, and Felix Frey, Brigitte Frey, and Matthias Ochs for the helpful discussions.

\section{References}

1. Caduff JH, Fischer LC, Burri PH: Scanning electron microscope study of the developing microvasculature in the postnatal rat lung. Anat Rec 1986, 216:154-164

2. Burri PH, Tarek MR: A novel mechanism of capillary growth in the rat pulmonary microcirculation. Anat Rec 1990, 228:35-45

3. Djonov V, Baum O, Burri PH: Vascular remodelling by intussusceptive angiogenesis. Cell Tissue Res 2003, 314:107-117
4. Burri PH, Hlushchuk R, Djonov V: Intussusceptive angiogenesis: its emergence, its characteristics, and its significance. Dev Dyn 2004, 231:474-488

5. Makanya AN, Hlushchuk R, Djonov VG: Intussusceptive angiogenesis and its role in vascular morphogenesis, patterning, and remodeling. Angiogenesis 2009, 12:113-123

6. Makanya AN, Stauffer D, Ribatti D, Burri PH, Djonov V: Microvascular growth, development, and remodeling in the embryonic avian kidney: the interplay between sprouting and intussusceptive angiogenic mechanisms. Microsc Res Tech 2005, 66:275-288

7. Makanya AN, Hlushchuk R, Baum O, Velinov N, Ochs M, Djonov V: Microvascular endowment in the developing chicken embryo lung. Am J Physiol Lung Cell Mol Physiol 2007, 292:1136-1146

8. Bagchus WM, Hoedemaeker PJ, Rozing J, Bakker WW: Acute glomerulonephritis after intravenous injection of monoclonal anti-thymocyte antibodies in the rat. Immunol Lett 1986, 12:109-113

9. Iruela-Arispe L, Gordon K, Hugo C, Duijvestijn AM, Claffey KP, Reilly M, Couser WG, Alpers CE, Johnson RJ: Participation of glomerular endothelial cells in the capillary repair of glomerulonephritis. Am J Pathol 1995, 147:1715-1727

10. Shimizu A, Masuda $Y$, Kitamura H, Ishizaki M, Sugisaki Y, Yamanaka $\mathrm{N}$ : Recovery of damaged glomerular capillary network with endothelial cell apoptosis in experimental proliferative glomerulonephritis. Nephron 1998, 79:206-214

11. Notoya M, Shinosaki T, Kobayashi T, Sakai T, Kurihara H: Intussusceptive capillary growth is required for glomerular repair in rat Thy-1.1 nephritis. Kidney Int 2003, 63:1365-1373

12. Ichimura K, Kurihara H, Sakai T: Involvement of mesangial cells expressing alpha-smooth muscle actin during restorative glomerular remodeling in Thy-1.1 nephritis. J Histochem Cytochem 2006, 54: 1291-1301

13. Wood JM, Bold G, Buchdunger E, Cozens R, Ferrari S, Frei J, Hofmann F, Mestan J, Mett H, O'Reilly T, Persohn E, Rösel J, Schnell C, Stover D, Theuer A, Towbin H, Wenger F, Woods-Cook K, Menrad A, Siemeister G, Schirner M, Thierauch KH, Schneider MR, Drevs J, Martiny-Baron G, Totzke F: PTK787/ZK 222584, a novel and potent inhibitor of vascular endothelial growth factor receptor tyrosine kinases, impairs vascular endothelial growth factor-induced responses and tumor growth after oral administration. Cancer Res 2000, 60: $2178-2189$

14. Hlushchuk R, Riesterer O, Baum O, Wood J, Gruber G, Pruschy M, Djonov $V$ : Tumor recovery by angiogenic switch from sprouting to intussusceptive angiogenesis after treatment with PTK787/ZK222584 or ionizing radiation. Am J Pathol 2008, 173:1173-1185

15. Carmeliet P: Angiogenesis in health and disease. Nat Med 2003, 9:653-660

16. Tuffin G, Waelti E, Huwyler J, Hammer C, Marti HP: Immunoliposome targeting to mesangial cells: a promising strategy for specific drug delivery to the kidney. J Am Soc Nephrol 2005, 16:3295-3305

17. Nyengaard JR: Stereologic methods and their application in kidney research. J Am Soc Nephrol 1999, 10:1100-1123

18. Smoyer WE, Mundel P, Gupta A, Welsh MJ: Podocyte alpha-actinin induction precedes foot process effacement in experimental nephrotic syndrome. Am J Physiol 1997, 273:150-157

19. Byzova TV, Goldman CK, Pampori N, Thomas KA, Bett A, Shattil SJ, Plow EF: A mechanism for modulation of cellular responses to VEGF: activation of the integrins. Mol Cell 2000, 6:851-860

20. Plate KH, Breier G, Risau W: Molecular mechanisms of developmental and tumor angiogenesis. Brain Pathol 1994, 4:207-218

21. Flyvbjerg A, Schrijvers BF, De Vriese AS, Tilton RG, Rasch R: Compensatory glomerular growth after unilateral nephrectomy is VEGF dependent. Am J Physiol Endocrinol Metab 2002, 283:362-366

22. Takahashi T, Huynh-Do U, Daniel TO: Renal microvascular assembly and repair: power and promise of molecular definition. Kidney Int 1998, 53:826-835

23. Reinders ME, Rabelink TJ, Briscoe DM: Angiogenesis and endothelial cell repair in renal disease and allograft rejection. J Am Soc Nephrol 2006, 17:932-942

24. Ostendorf $T$, Kunter $U$, Eitner $F$, Loos A, Regele $H$, Kerjaschki D, Henninger DD, Janjic N, Floege J: VEGF (165) mediates glomerular endothelial repair. J Clin Invest 1999, 104:913-923

25. Kang DH, Hughes J, Mazzali M, Schreiner GF, Johnson RJ: Impaired angiogenesis in the remnant kidney model, II: vascular endothelial 
growth factor administration reduces renal fibrosis and stabilizes renal function. J Am Soc Nephrol 2001, 12:1448-1457

26. Malmström NK, Kallio EA, Rintala JM, Nykänen Al, RäisänenSokolowski AK, Paavonen T, Lemström KB, Koskinen PK: Vascular endothelial growth factor in chronic rat allograft nephropathy. Transpl Immunol 2008, 19:136-144

27. Flyvbjerg A, Dagnaes-Hansen F, De Vriese AS, Schrijvers BF, Tilton RG, Rasch R: Amelioration of long-term renal changes in obese type 2 diabetic mice by a neutralizing vascular endothelial growth factor antibody. Diabetes 2002, 51:3090-3094

28. Schrijvers BF, Flyvbjerg A, Tilton RG, Lameire NH, De Vriese AS: A neutralizing VEGF antibody prevents glomerular hypertrophy in a model of obese type 2 diabetes, the Zucker diabetic fatty rat. Nephrol Dial Transplant 2006, 21:324-329

29. Floege J, Ostendorf T, Janssen U, Burg M, Radeke HH, Vargeese C, Gill SC, Green LS, Janjić N: Novel approach to specific growth factor inhibition in vivo: antagonism of platelet-derived growth factor in glomerulonephritis by aptamers. Am J Pathol 1999, 154:169-179

30. Floege J, Eitner F, Alpers CE: A new look at platelet-derived growth factor in renal disease. J Am Soc Nephrol 2008, 19:12-23

31. Boor $P$, van Roeyen CR, Kunter U, Villa L, Bücher E, Hohenstein B, Hugo CP, Eriksson U, Satchell SC, Mathieson PW, Eitner F, Floege J, Ostendorf T: PDGF-C mediates glomerular capillary repair. Am J Pathol 2010, 177:58-69

32. Inai $T$, Mancuso $M$, Hashizume $H$, Baffert $F$, Haskell $A$, Baluk $P$, Hu-Lowe DD, Shalinsky DR, Thurston G, Yancopoulos GD, McDonald DM: Inhibition of vascular endothelial growth factor (VEGF) signaling in cancer causes loss of endothelial fenestrations, regression of tumor vessels, and appearance of basement membrane ghosts. Am J Pathol 2004, 165:35-52

33. Kamba T, Tam BY, Hashizume H, Haskell A, Sennino B, Mancuso MR, Norberg SM, O'Brien SM, Davis RB, Gowen LC, Anderson KD, Thurston G, Joho S, Springer ML, Kuo CJ, McDonald DM: VEGFdependent plasticity of fenestrated capillaries in the normal adult microvasculature. Am J Physiol Heart Circ Physiol 2006, 290 560-576

34. Fayard E, Tintignac LA, Baudry A, Hemmings BA: Protein kinase B/Akt at a glance. J Cell Sci 2005, 118:5675-5678

35. Johnson RJ, lida H, Alpers CE, Majesky MW, Schwartz SM, Pritzi P, Gordon K, Gown AM: Expression of smooth muscle cell phenotype by rat mesangial cells in immune complex nephritis: alpha-smooth muscle actin is a marker of mesangial cell proliferation J Clin Invest 1991 87:847-858

36. Johnson RJ, Floege J, Yoshimura A, lida H, Couser WG, Alpers CE The activated mesangial cell: a glomerular "myofibroblast"? J Am Soc Nephrol 1992, 2(10 Suppl):S190-S197

37. Morioka T, Yao J, Suzuki Y, Oite T: The characterization of a specific Thy-1 molecular epitope expressed on rat mesangial cells. Kidney Int 2004, 66:2214-2223

38. Takahara H, Shirato I, Asanuma K, Yamashita M, Takeda Y, Tomino Y: Tensin is expressed in glomerular mesangial cells and is related to their attachment to surrounding extracellular matrix. J Histochem Cytochem 2004, 52:683-691

39. Satchell SC, Braet F: Glomerular endothelial cell fenestrations: an integral component of the glomerular filtration barrier. Am J Physiol Renal Physiol 2009, 296:947-956

40. Watanabe S, Yoshimura A, Inui K, Yokota N, Liu Y, Sugenoya Y, Morita $\mathrm{H}$, Ideura $\mathrm{T}$ : Acquisition of the monocyte/macrophage phenotype in human mesangial cells. J Lab Clin Med 2001, 138:193199

41. Langenberg $\mathrm{MH}$, Witteveen $\mathrm{PO}$, Lankheet NA, Roodhart JM, Rosing $\mathrm{H}$, van den Heuvel IJ, Beijnen JH, Voest EE: Phase 1 study of combination treatment with PTK 787/ZK 222584 and cetuximab for patients with advanced solid tumors: safety, pharmacokinetics, pharmacodynamics analysis. Neoplasia 2010, 12:206-213

42. Banerjee S, Zvelebil M, Furet $P$, Mueller-Vieira U, Evans DB, Dowsett $M$, Martin LA: The vascular endothelial growth factor receptor inhibitor PTK787/ZK222584 inhibits aromatase. Cancer Res 2009, $69: 4716-4723$ 